\title{
Commentary
}

\section{First Heal Thyself}

\section{Rescue of Dysfunctional Endothelial Progenitor Cells Restores Function to the Injured Kidney}

\author{
Leon G. Fine \\ From the Cedars-Sinai Medical Center, Department of \\ Biomedical Sciences, Los Angeles, California
}

While the attention of the scientific community is focused on a panoply of possibilities for the use of progenitor and stem cells in promoting repair in a variety of tissues and organs, a study appears which suggests that these cells themselves may need to undergo repair before they can mount an effective healing and regenerative response. In this issue of the Journal, Yasuda and coworkers outline a convincing case for resident endothelial progenitor cell incompetence in the injured kidney as well as showing that this can be overcome by adoptive transfer of cells of the same type. ${ }^{1}$

The backdrop of this intriguing study was provided by the work of Challen et al ${ }^{2}$ who followed others ${ }^{3-5}$ in characterizing a "kidney side population" of cells, demonstrating that injection of these cells was able to reduce proteinuria in a mouse model of adriamycin-nephropathy. These data built on studies of murine hematopoietic stem cells, which demonstrate low fluorescence after staining with the dye Hoechst 33342, a characteristic which has been useful in the purification of these cells. When used in combination with other markers, such low-dye-expressing cells have been described as "side population" cells based on their ability to export intracellular dye rapidly. ${ }^{5-7}$ Putative stem cells from a variety of tissues appear to conform to this phenotype. ${ }^{8}$

In the study of Challen et al, ${ }^{2}$ a resident population of cells of nonhematopoietic immunotype was identified, with a proximal tubular location and the ability to differentiate into multiple lineages. Introduction of such cells into mice with adriamycin-nephropathy improved glomerular filtration rates and decreased albuminuria but did not appear to integrate into renal tubules, leaving the mechanism for functional improvement unclear.

The present study of Yasuda et al has taken this story forward in a new and potentially exciting direction. Using the adriamycin-nephropathy model, they hypothesized that impairment of endothelial progenitor cell (EPC) function is the basis for impaired regeneration and, if this were the case, rescue by exogenous endothelial precursor cells might be possible. They analyzed hematopoietic stem cells (HSCs) and EPCs derived from the kidneys of mice with adriamycin-nephropathy. (HSCs were CD150 and CD117 [c-kit] double-positive, and EPCs were CD34 and Flk-1 double-positive.)

Both resident populations were sparse $(<0.1 \%$ of total cell number) in control kidneys and did not change numerically after adriamycin-induced injury. These cells demonstrated decreased viability, increased senescence, and increased apoptosis, which were not attributable to loss of stromal cell-derived factor-1 production, a potential "niche" for such cells. In other words, they were dysfunctional cells.

Adoptive transfer of normal EPCs resulted in improvement of glomerular filtration rate and reduction in proteinuria with a threefold decrease in mortality. This appeared to be associated with an improvement in the density of the microvasculature and a reduction of apoptosis, which were paralleled by reduced plasma levels of interleukin- $1 \alpha$ and $-\beta$ and Granulocyte Colony-Stimulating Factor (G-CSF) and an increase in vascular endothelial growth factor.

A single injection of EPCs led to an approximately sevenfold increase in both HSCs and EPCs in the diseased kidneys, representing less than $2 \%$ of the injected cells. (The time course of the EPC increase in the kidney suggested that trafficking via the bone marrow may occur.)

One route by which these adoptively-transferred cells were able to engage the resident cell population, and which was explored by Yasuda et $\mathrm{al}^{1}{ }^{1}$ was tunneling nanotube formation between EPCs and mature endothelial cells. The formation of these microscopic tube-like structures extending between cells allows for the transfer

Accepted for publication January 12, 2010.

Address reprint requests to Leon G. Fine, M.D., Cedars-Sinai Medical Center, Department of Biomedical Sciences, 8700 Beverly Boulevard, Davis Building \#5072, Los Angeles, CA 90048. E-mail: leon.fine@cshs.org. 
of subcellular organelles. Transfer of fluorescent mitochondria was demonstrated from EPCs to adriamycininjured HUVECs in vitro.

Adoptive transfer of EPCs (which is achieved by systemic administration and does not require direct injection into the kidney) may serve as a useful tool, or even therapy, for mobilizing a healing response in diseased organs, but the route by which this may be achieved is not clear; what is intriguing is the possibility of a central role played by the microvasculature in the process.

There is now convincing evidence in a number of experimental models of progressive kidney disease, that chronic hypoxia, which is associated with interstitial microvascular insufficiency and obliteration, is central to the progressive scarring process. ${ }^{9,10}$ The possibility exists that homing of progenitor cells could be augmented by local hypoxia, ${ }^{9}$ but there is no current information to support or refute this. Evidence that adriamycin-nephropathy is associated with a decrease in glomerular and peritubular capillary density ${ }^{1}$ indicates that, if left untreated, the kidneys will undergo scarring and loss of function. The restoration of microvasculature density with increased plasma vascular endothelial growth factor levels after adoptive transfer of EPC, in the study of Yasuda et al, ${ }^{1}$ points to the potential reversibility of such kidney injury.

The debate continues about whether or not a "renal stem cell" exists, and, if such a cell-type does exist, could it be used in a cellular therapeutic context? The literature remains conflicting on this issue. ${ }^{11}$ The fact that the kidney has the ability to recover from acute injury does not imply the existence of such cells because differentiated resident cells could regenerate themselves. What seems quite certain, however, is that it is not possible to re-grow new nephrons in the adult kidney. So, any repair which does occur would have to be within the scaffolding of an existing nephron structure. Could a progenitor cell that gives rise to cells of different phenotypes achieve this feat by generating different phenotypes in different segments of the adult nephron? Although possible, this scenario is hard to imagine.

At the end of the day, the architecture of the kidney makes it very different from other organs, such as the liver or the gastrointestinal tract, where cellular proliferation is the predominant repair mechanism. In contrast, each microscopic nephron is a home unto itself, and cellular regeneration and hypertrophy must occur within the confines of its existing walls.

An intriguing possibility not discussed in the paper of Yasuda et al is that reversal of a variety of kidney diseases and progressive diseases of other organs may not require that stem cells be differentiated into phenotypes specific to the organ in order to achieve healing and regeneration, but that a strategy that results only in microvasculature restoration could serve as the common pathway to healing. Certainly in an acute setting, selective engraftment of a kidneyderived mesenchymal cell line has been shown to result in vasculogenesis and to promote functional recovery after acute ischemic injury of the kidney. ${ }^{12}$

So, a new scenario suggests itself. It could be argued that the only prerequisite for renal regeneration after chronic injury is an adequate microvasculature supply and that it is the job of endothelial progenitor cells to look after this. When the kidney is injured, for instance by adriamycin, such cells malfunction and the microvasculature deteriorates in parallel with dysfunction of endothelial precursors. The ensuing local hypoxia leads to nephron loss and organ fibrosis. To reverse this process, all that may be needed is to re-establish normal EPC function or to re-supply normally functioning EPCs. Such normally-functioning progenitor cells could link up with resident endothelial cells to re-establish a functioning microvasculature. Improved perfusion and oxygenation would allow for the resident tubular and glomerular cells on the margins of injured areas to undergo hyperplasia and repair within the scaffolding of surviving nephrons. No tubular or glomerular cell differentiation from progenitor cells is needed for such repair, and no new nephrons need to be created. A restored microvasculature may be all that is required to initiate a fully differentiated tissue response to restore chronically-injured tissue. The kidney could then breathe again! The same paradigm could apply to the regeneration of other organs.

\section{References}

1. Yasuda K, Park H, Ratliff B, Addabbo F, Hatzopoulos AK, Chander P Goligorsky MS: Adriamycin nephropathy - A failure of endothelial progenitor cell-induced repair. Am J Pathol 2010, 176:1685-1695

2. Challen GA, Bertoncello I, Deane JA, Ricardo SD, Little MH: Kidney side population reveals multilineage potential and renal functional capacity but also cellular heterogeneity. J Am Soc Nephrol 2006, 17:1896-1912

3. Asakura A, Rudnicki MA: Side population cells from diverse adult tissues are capable of in vitro hematopoietic differentiation. Exp Hematol 2002, 30:1339-1345

4. Hishikawa K, Marumo T, Miura S, Nakanishi A, Matsuzaki Y, Shibata K, Ichiyanagi T, Kohike H, Komori T, Takahashi I, Takase O, Imai N, Yoshikawa M, Inowa T, Hayashi M, Nakaki T, Nakauchi H, Okano H, Fujita T: Musculin/MyoR is expressed in kidney side population cells and can regulate their function. J Cell Biol 2005, 169:921-928

5. Iwatani H, Ito T, Imai E, Matsuzaki Y, Suzuki A, Yamato M, Okabe M, Hori M: Hematopoietic and nonhematopoietic potentials of Hoechst(low)/side population cells isolated from adult rat kidney. Kidney Int 2004, 65:1604-1614

6. Schinkel AH, Mayer U, Wagenaar E, Mol CA, van Deemter L, Smit JJ, van der Valk MA, Voordouw AC, Spits $H$, van Tellingen O, Zijlmans JM, Fibbe WE, Borst P: Normal viability and altered pharmacokinetics in mice lacking mdr1-type (drug-transporting) P-glycoproteins. Proc Natl Acad Sci USA 1997, 94:4028-4033

7. Zhou S, Schuetz JD, Bunting KD, Colapietro AM, Sampath J, Morris JJ, Laguntina I, Grosveld GC, Osawa M, Nakauchi H, Sorrentino BP: The ABC transporter Bcrp1/ABCG2 is expressed in a wide variety of stem cells and is a molecular determinant of the side-population phenotype. Nat Med 2001, 7:1028-1034

8. Challen GA, Little MH: A side order of stem cells: the SP phenotype. Stem Cells 2006, 24:3-12

9. Fine LG, Norman JT: Chronic hypoxia as a mechanism of progression of chronic kidney diseases: from hypothesis to novel therapeutics. Kidney Int 2008, 74:867-872

10. Nangaku M, Inagi R, Miyata T, Fujita T: Hypoxia and hypoxia-inducible factor in renal disease. Nephron Experimental Nephrology 2008, 110:e1-e7

11. Little MH, Bertram JF: Is there such a thing as a renal stem cell? J Am Soc Nephrol 2009, 20:2112-2117

12. Chen J, Park H, Addabbo F, Ni J, Pelger E, Li H, Plotkin M, Goligorsky MS: Kidney-derived mesenchymal stem cells contribute to vasculogenesis, angiogenesis and endothelial repair. Kidney Int 2008, 74:879-889 